\title{
An understanding of muscle fibre images
}

\author{
C.C. Taylor, M.R. Faghihi and I.L. Dryden \\ Department of Statistics, University of Leeds, Leeds LS2 9JT, U.K.
}

\begin{abstract}
Images of muscle biopsies reveal a mosaic pattern of two (slow-twitch and fast-twitch) fibre-types. An analysis of such images can indicate some neuromuscular disorder. We briefly review some methods which analyse the arrangement of the fibres (e.g. clustering of fibre type) and the fibre sizes. The proposed methodology uses the cell centres as a set of landmarks from which a Delaunay triangulation is created. The shapes of these (correlated) triangles are then used in a test statistic, to ascertain normality of a muscle. Our "normal muscle" model supposes that the fibres are hexagonal (so that the triangulation is made up of equilateral triangles) with a perturbation of specified isotropic variance of the fibre centres. We obtain the distribution of the test statistic as an approximate function of a $\chi^{2}$ random variable, so that a formal test can be carried out.
\end{abstract}

\section{Introduction}

An important component in the early diagnosis of muscle diseases is the ability to (automatically) analyse muscle biopsies. Using either the needle biopsy technique (Edwards, 1971), the open surgical technique (Dubowitz, 1985) or the semi-open technique (Henriksson, 1979), part of several hundred fibres can be extracted for examination. These cross-sections are usually histochemically stained to reveal a light microscopic examination of two type of fibres: type 1 (lightly stained) and type 2 (heavily stained). Figure 1 shows two examples (at different magnifications) of a small part of images obtained from whole muscle cross-sections (obtained post-mortem), but similar images would also result from biopsies. There are important changes that occur with age, for example a decrease in muscle area and a decrease in fibre size; see Lexell et al. (1988) for details. Deviations from normality, and hence indicating some neuromuscular disorder, can occur in a variety of ways. For example, the formation of large groups of fibres of the same histochemical type is considered to be evidence of a denervation and reinervation process (Walton, 1981; Dubowitz, 1985), and a variety of objective methods have been suggested to detect such grouping: see Lexell, Downham \& Sjöstrom (1987) for a review of statistical approaches.

An analysis of fibre size has been investigated from fibre outlines digitised by hand on a digitising tablet. This enables an investigation of the difference in the distribution between type I and type II fibres within a given region of the muscle, and differences in distributions between different parts of the muscle, for example superficial fibres tend to have less variability. This difference may be expressed in terms of the mean and/or variance, but more often there are important features in the distribution, such as evidence of "subgroups", which are most easily seen in a nonparametric density estimate. These can be readily superimposed, as an example shows in Figure 2. 

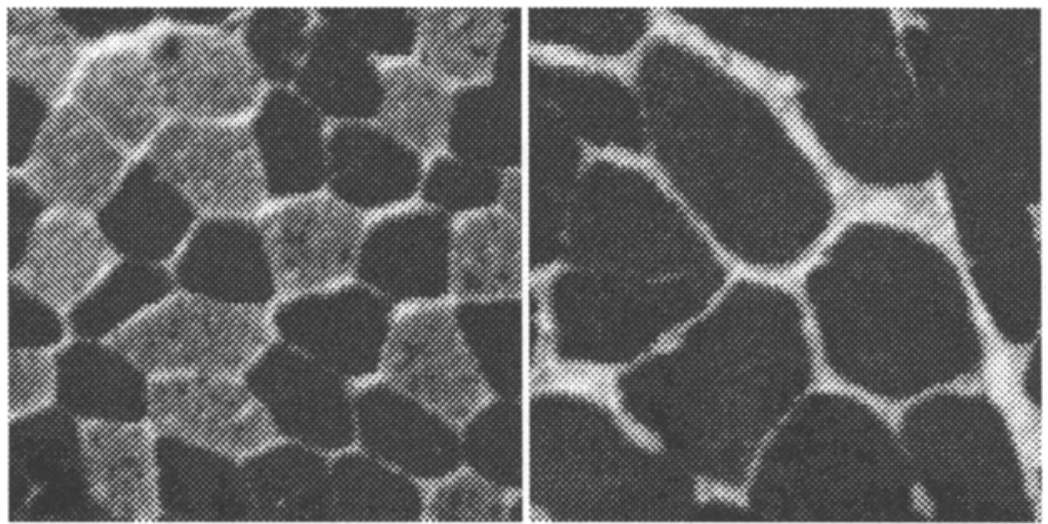

Fig. 1. Examples from a normal muscle. Left: the fibres are fairly hexagonal in shape and apparently quite random in type, Right: close-up shows some angular shaped fibres
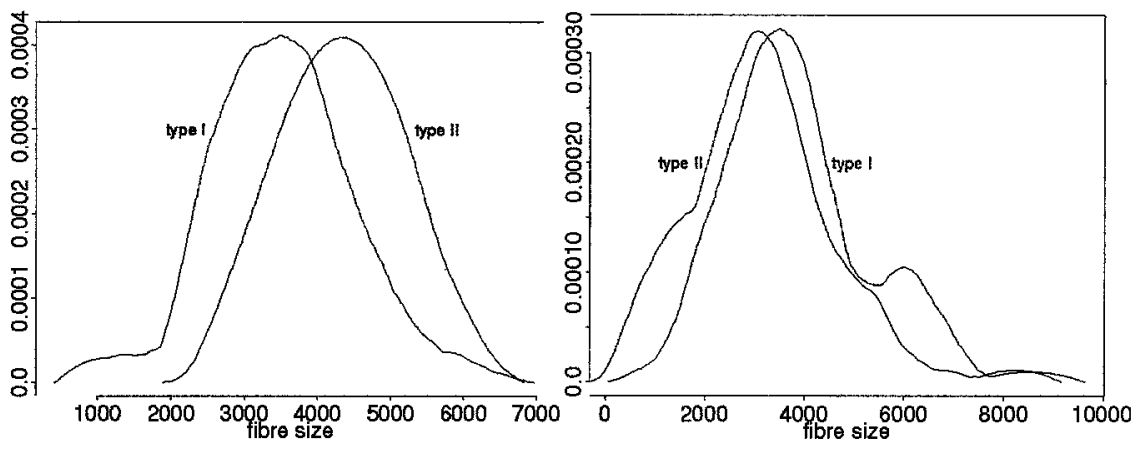

Fig. 2. Normal muscle examples. Note the sub-populations indicated by bimodality.

Hand digitisation is a laborious process, and a system which can segment each fibre and label it as type I or type II would capture all the essential information for an objective classification of each muscle biopsy. One difficulty in segmentation arises from grey-level trends in which the type of fibre can only be determined from the relative brightness. This could be dealt with by subtraction of a smoothed background. More importantly, detecting an edge between two abutting fibres of the same type can be very difficult. In principle, it should be possible to use prior information that fibres are usually convex in shape. A Bayesian approach, which attaches a probability distribution to each fibre shape (and size) could then give a more robust segmentation. A less time consuming digitisation would be to mark the "centres" (for example, the approximate centroid) of each fibre, together with the fibre type. This takes only a few minutes for each region studied (about 150 fibres). 


\section{Shape}

Healthy muscles generally contain fibres which are roughly hexagonal in shape, so an analysis of fibre shape may detect an abnormality. However, in view of the above remarks on ease of digitisation we instead study the "shape" of the marked centres. The methodology that has been developed to date does not use the information in the fibre type, so we initially throw away the labelling.

\subsection{Landmarks and Triangulation}

Shape analysis is often based on a set of "landmarks" i.e. points of an object (or image) which are easily identified. This set of co-ordinates $\left\{\left(x_{i}, y_{i}\right), i=1,2, \ldots, n\right\}$ is then transformed to take out the effects of scale, rotation and translation. The information which remains is thus referred to as shape. In the current context we use the centres of the fibres as our set of landmarks, and we begin by forming a Delaunay triangulation (see Figure 3). We first associate, for each $\left(x_{i}, y_{i}\right)$, the polygon $T\left(x_{i}, y_{i}\right)$ which is the set of points for which $\left(x_{i}, y_{i}\right)$ is the nearest neighbour amongst all landmarks. These polygons, known as Voronoi polygons form a Dirichlet tessellation of the data, and they have the property that almost surely, except in the case of certain regular structures, every node is touched by exactly three cells. The Delaunay triangulation is constructed by connecting those landmarks whose cells share the same node. Note that an edge correction procedure is required in which cells which lie on the boundary of the image are removed in order to avoid flat "splinter like" triangles.

\subsection{Summary statistic}

The proposed model is that a normal muscle has fibres which are close to hexagonal in shape, in which case the Voronoi polygons associated with the fibre centres are just the fibres themselves, and the associated Delaunay triangles are equilateral. So we suppose that normal fibres could be simulated with centres $\left(X_{i}, Y_{i}\right)$ with distribution

$$
X_{i} \sim N\left(\alpha_{i}, \sigma^{2}\right), \quad Y_{i} \sim N\left(\beta_{i}, \sigma^{2}\right), \quad i=1,2, \ldots, n
$$

all mutually independent, where the $\left(\alpha_{i}, \beta_{i}\right), i=1,2, \ldots, n$ are chosen such that their associated Delaunay triangulation contains equal sized equilateral triangles; see Mardia at al. (1977). The value of $\sigma$ then gives a deviation from the regular (hexagonal) case; a large $\sigma$ emulating a diseased muscle, and a small $\sigma$ indicating a healthy muscle. Figure 3 has two realisations for different values of $\sigma$.

Let $\delta$ be the length of the edge of each equilateral triangle. As shape is independent of the scale of the image we examine whether or not $\tau=\sigma / \delta$ is large. Estimation of $\tau$ for some real data then gives a plausible test statistic which can be used to test $H_{0}: \tau=\tau_{0}$ vs $H_{1}: \tau>\tau_{0}$ if we have a given $\tau_{0}$ which has been previously estimated from known healthy muscles. However, in practice, it is easier to work with the shape of the triangles, rather than estimate $\tau$ directly. Define the shape distance between two triangles $\left(\left(x_{i}, y_{i}\right)\right.$ and $\left.\left(\alpha_{i}, \beta_{i}\right), i=1,2,3\right)$ as $\rho$ where

$$
(\cos \rho)^{2}=\frac{\left(S_{x \alpha}+S_{y \beta}\right)^{2}+\left(S_{x \beta}-S_{y \alpha}\right)^{2}}{\left(S_{x x}+S_{y y}\right)\left(S_{\alpha \alpha}+S_{\beta \beta}\right)}
$$




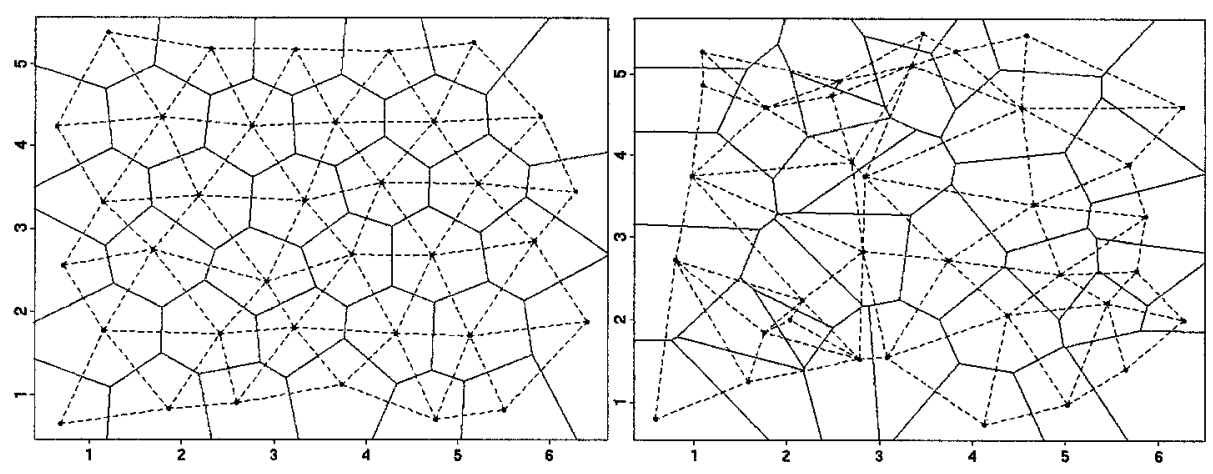

Fig. 3. Examples of Dirichlet tessellation (solid) and Delaunay triangulation (dashed). Left: $\sigma=0.1$; Right: $\sigma=0.3$

with

$$
S_{x y}=\sum_{i=1}^{3}\left(x_{i}-\bar{x}\right)\left(y_{i}-\bar{y}\right), \quad \bar{x}=\frac{1}{3} \sum_{i=1}^{3} x_{i}, \quad \bar{y}=\frac{1}{3} \sum_{i=1}^{3} y_{i} .
$$

The distance is known as Riemannian distance between two triangle shapes and was introduced by Kendall (1984). We can regard $\rho$ as a measure of similarity between two triangles, after removing location, rotation and scale effects.

\subsection{Distribution}

It can be shown (see Dryden et al., 1995 for details) that, for small $\tau=\sigma / \delta$

$$
\cos \rho \sim 1-\frac{\tau^{2}}{2} \chi_{2}^{2}
$$

where $\chi_{2}^{2}$ represents a Chi-squared random variable with 2 degrees of freedom. This work is related to previous work on perturbations in Procrustes methods; see Sibson (1978, 1979), Langron \& Collins (1985), Goodall (1991) and Mardia (1989). Suppose now that we have $n$ landmarks which will form $m$ triangles. Note that the shape of neighbouring triangles are correlated, so the distribution of

$$
\overline{\cos \rho}=\frac{1}{m} \sum_{l=1}^{m} \cos \rho_{l}
$$

must take account of the neighbourhood structure. In principle it would be possible to use simulation to obtain results, for example, to give $p$-values for a certain null hypothesis. However, it is straightforward to obtain the approximate distribution

$$
\overline{\cos \rho} \sim 1-\frac{\tau^{2}}{4 m} \sum_{i=1}^{n} \lambda_{i} \chi_{2}^{2}
$$


where the $\lambda_{i} \geq 0$ are eigenvalues of a matrix associated with the contiguity structure of the $\left(\alpha_{i}, \beta_{i}\right)$. By matching the mean and variance we can further approximate this linear sum of $\chi^{2}$ random variables to get

$$
\overline{\cos \rho} \sim 1-a \chi_{\nu}^{2}
$$

with

$$
\nu=\frac{8 m^{2}}{\sum_{i=1}^{n} \lambda_{i}^{2}}, \quad a=\frac{\tau^{2}}{\nu}
$$

Exact values of $\sum \lambda_{i}^{2}$ can be determined for a given sample, but we can usually use the approximation that, for a rectangular array of $m_{1} \times m_{2}$ triangles, we have

$$
\sum_{i=1}^{n} \lambda_{i}^{2}=\frac{4}{9}\left(21 m_{1} m_{2}-8 m_{2}-14 m_{1}+c\right)
$$

where $c$ can be 6,8 or 10 according to the different arrangements of the triangles. It is of interest to examine how the variance of the statistic, and hence $\sum \lambda_{i}^{2}$ varies for different choices of $\left(\alpha_{i}, \beta_{i}\right), i=1, \ldots, n$; see Dryden et al. (1995) for details.

\subsection{Hypothesis Testing}

If we have only one image, then it is straightforward to compute $\overline{\cos \rho}$ which then gives an estimate of $\tau$ as

$$
\hat{\tau}=\sqrt{1-\overline{\cos \rho}}
$$

We can then test $H_{0}: \tau=\tau_{0}$ vs $H_{1}: \tau>\tau_{0}$ by using the test statistic

$$
T=\frac{\nu}{\tau_{0}^{2}}(1-\overline{\cos \rho}) \sim \chi_{\nu}^{2}
$$

if the null hypothesis is true.

If we have multiple images (from the same individual, or from a group of individuals from the same class) then we can estimate $\tau$ by maximum likelihood and proceed in the usual way. In principle, $\tau_{0}$ can be estimated from known healthy muscles and used as a subsequent benchmark.

Example Suppose that the null hypothesis of regularity is $H_{0}: \tau \leq 0.15$, and that the alternative hypothesis of irregularity is $H_{1}: \tau>0.15$. Suppose that the image of muscle fibres has $m=176$ in a roughly rectangular region (with $m_{1}=11, m_{2}=16$ ), and that $\overline{\cos \rho}=0.9711$. Then we have $\nu=163$ and test statistic

$$
T=\frac{163}{0.15^{2}}(0.0289)=209.4 \sim \chi_{163}^{2}
$$

if $H_{0}$ is true. For large $\nu$ we can use the Normal approximation $\sqrt{2 T}-\sqrt{2 \times 163-1} \sim$ $N(0,1)$. This gives a $p$-value of about 0.0149 , so we reject $H_{0}$. 


\section{Extensions}

The above triangulation can also be used to describe the size of the fibres, $s$, given by

$$
3 s^{2}=S_{x x}+S_{y y}
$$

and in a similar manner we can obtain a test statistic for the variance of triangle sizes.

A further extension is to use the labels of the landmarks (type I or type II). We can then either consider the distribution of the 4 types of triangle, i.e. those which have $0,1,2$ or 3 type I vertices, or we can obtain a separate triangulation for each label, and examine the size and shape of these triangles. Both of these approaches may usefully combine information in size, shape and arrangement of fibre type colours, and lead to powerful discrimination methods.

Acknowledgement: The authors would like to thank Jan Lexell, University of Lund for providing the muscle fibre images, and introducing us to this problem.

\section{References}

Goodall, C.: Procrustes methods in the statistical analysis of shape. Journal of Royal Statistical Society B, 53 285-339 (1991)

Dubowitz, V.: Muscle biopsy. A practical. Baillière Tindal, London (1985)

Edwards, R.H.T.: Percunatenous needle biopsy of skeletal muscle in diagnosis and research. Lancet, 11 593-596 (1971)

Dryden, I.L., Faghihi, M.R. and Taylor, C.C.: Shape analysis of spatial point patterns. In: K.V. Mardia, C.A. Gill (eds.): Proceedings in Current Issues in Statistical Shape Analysis. Leeds University Press 1995, pp. 40-48.

Henriksson, K.G.: "Semi-open" muscle biopsy technique. A simple outpatient procedure. Acta Neurol Scand 59 317-323 (1979)

Kendall, D.G.: Shape - manifolds, procrustean metric and complex projective space. Bull. Lond. Math. Soc. 16 81-121 (1984)

Langron, S.P. and Collins, A.J.: Perturbation theory for generalized Procrustes analysis Journal of the Royal Statistical Society B 47 277-284 (1985)

Lexell, J., Downham, D. and Sjöstrom, M.: Morphological detection of neurogenic muscle disorders: how can statistical methods aid diagnosis? Acta Neuropathol 75 109115 (1987)

Lexell, J., Taylor, C.C. and Sjöstrom, M.: What is the cause of the ageing atrophy? Total number, size and proportion of different fiber types studied in whole vastus lateralis muscle from 15- to 83-year-old men. J. Neurol. Sci. 84 275-294 (1988)

Mardia., K. V.: Shape analysis of triangles through directional techniques. Journal of Royal Statistical Society B 51 449-458 (1989)

Mardia, K.V., Edwards, R. and Puri, M.L. Analysis of Central Place Theory. Bulletin of the International Statistical Institute 47 93-110 (1977)

Sibson, R. (1978) Studies in the robustness of multidimensional scaling: Procrustes statistics.

Sibson, R.: Studies in the robustness of multidimensional scaling: Procrustes statistics. Journal of the Royal Statistical Society, B 40 234-238 (1978)

Sibson, R.: Studies in the robustness of multidimensional scaling: perturbation analysis of classical scaling. Journal of the Royal Statistical Society, B 41 217-229 (1979)

Walton, J.: Disorders of Voluntary Muscle. London: Churchill Livingston 1981 